\title{
The Anthropomorphic Robot Arm Joint Control Parameter Tuning Based on Ziegler Nichols PID
}

\author{
Renli WANG ${ }^{1, a}$, Yueming DAl ${ }^{2, b}$ \\ ${ }^{1}$ Department of Information and Electric Engineering, Xuzhou Institute of Technology, Xuzhou, \\ 221111, China \\ 2 Department of Information and Electric Engineering, Xuzhou Institute of Technology, Xuzhou, \\ 221111, China \\ aemail: renliwang@163.com, bemail: daiym63@163.com
}

Keywords: Anthropomorphic robot; Ziegler and Nichols parameter tuning; multi-joint control; Critical stable

\begin{abstract}
In order to get the anthropomorphic robot accurate parameters of the upper limb joint control motor, a method based on Ziegler Nichols-frequency response of the adaptive PID controller is proposed. The control circuit identifies online important critical frequency response characteristics of process object through input and output data in the normal process operation, and then the system online update PID controller parameters based on Ziegler-Nichols setting rules or by the improved method. The results show that the method has stronger robustness and adaptive ability. It can always guarantee control circuit run in the best condition and almost completely resistant to outside interference. The simulation is not only an important guiding role to the design of anthropomorphic robot hand control node, but also certain reference significance for the dc motor closed-loop control.
\end{abstract}

\section{Introduction}

Anthropomorphic robot hand control node's task [1][2][3]is to receive robot "brain" (PC) instruction and complete the hand motor speed and position control. Personification robot hand motor mostly adopted DC motor, anti-interference ability of which is poor usually. To overcome outside interference, decrease the speed fluctuation, obtain high control accuracy and response speed, we put forward the Ziegler Nichols [4][5]parameter setting method: when the process variables and speed change, we establish a set of empirical formula that transform these test results into the right performance set parameters. The Ziegler-Nichols frequency response method can online optimize mine hoist speed regulator parameters in direct torque control system, restrain the low speed torque ripple, and improve the control precision of the system. It can guarantee the control loop always run in the best state. By establishing frequency converter speed model, we constructed hoist control system simulation diagram based on PID Ziegler-Nichols setting method. The simulation results show that the PID controller based on Ziegler-Nichols PID setting method has good dynamic performance on tracking the set value response, less overshoot and good steady state performance. The system has a strong ability to adapt to the object parameters change.

\section{The Mathematical Model of the DC Motor}

The actual measurement curve of the motor and theoretical formula are compared, open loop system time response function of anthropomorphic of robot hand motor [6] [7] is:

$$
a \frac{d^{2} \omega(t)}{d t^{2}}+b \frac{d \omega(t)}{d t}+c \omega(t)=e_{i}(t)
$$

In the type: $\mathrm{a}=284.126 ; \mathrm{b}=1282.297 ; \mathrm{c}=0.084$.

$522284.126 \frac{d^{2} \omega(t)}{d t^{2}}+1282.297 \frac{d \omega(t)}{d t}+0.0843 \omega(t)=e_{i}(t)$ 
Join the input and output speed conversion link, to meet the system requirements [8]:

$$
G(s)=\frac{1}{522284.126 S^{2}+1282.297 S+0.0843}
$$

\section{The PID Controller Parameters Influence on Control Performance}

$$
\begin{aligned}
& \Delta u(k)=u(k)-u(k-1)=K_{P}[e(k)-e(k-1)]+K_{I} e(k)+K_{D}[e(k)-2 e(k-1)+e(k-2)] \\
& u(k)=K_{P}\left\{e(k)+\frac{T}{T_{I}} \sum_{j=0}^{k} e(j)+\frac{T_{D}}{T}[e(k)-e(k-1)]\right\}
\end{aligned}
$$

Formula (4) is called incremental PID control formula; Formula (5) is called position type PID control equation [8].

In the type: Kp is proportional coefficient, $K_{I}=K_{P} \frac{T}{T_{I}}$ is integral coefficient and $K_{D}=K_{P} \frac{T_{D}}{T}$ is differential coefficient.

\subsection{The comparison of two kinds of PID control algorithm}

Because position type PID controller adopts total output, its output is the actual position of the actuator, and each output is associated with the past state and calculation of deviation accumulation. So large amount of calculation work must run, once the data processing computing chips appear problem, the system will make the output volatility and result in high volatility in the actuator, which causes big accidents. The incremental PID algorithm is better.

In incremental algorithm, the proportional and integral item symbols have the following relations: if the controlled variables to continue deviates from the given value, the two symbols are the same; and when the controlled variables to the given value direction changes, the two symbols instead. This algorithm can make the dynamic process of control system accelerate.

\subsection{The PID controller parameters influence on control performance}

(1) Proportional coefficient $\mathrm{Kp}$ : the role is to accelerate the response speed of system and improve the adjustment accuracy of system. When the system once appeared deviation, proportional control will produce an immediate adjustment to reduce error. The greater the Kp, the faster the system response. But it will produce overshoot and oscillation even lead to system instability, so the value cannot select too much; But if the value is too small, it will reduce regulation accuracy and system response speed, then it prolong to adjust time and make the system static and dynamic characteristics worse.

(2) The integral coefficient Ki: its role is to eliminate the steady-state error of the system and improve the degree of no error. The bigger the Ki, the faster the static error is eliminated. But if it is too big, it can produce integral saturation phenomenon at the beginning of the response process, and cause a big overshoot to make the dynamic performance worse; But if the value is too small, it will make integral effect weakened, and the system static error is difficult to eliminate, so transition time is longer. The system can't reach a steady state as soon as possible, then affect the system control precision and dynamic characteristic.

(3)Differential coefficient $\mathrm{Kd}$ : its role is to improve the system dynamic characteristics, reflect the deviation signal change rate, and foresee the deviation change trend. It can produce advanced control function, reduce the overshoot of system, and increase the system stability. But it's not too much, Otherwise it not only make the response process braking ahead of time and prolong system adjustment time, but also make the anti-interference of the system worse.

The purpose of parameter setting is by adjusting the three parameters of PID Kp, Ki and Kd to make the closed-loop characteristic root of system distribute a particular domain in the left half plane of s domain, to ensure that the system has enough stability margin and satisfy the given performance target. 


\section{Ziegler Nichols Method to Realize the PID Parameter Setting}

Ziegler and Nichols method has developed online parameters setting between experience and calculation method. Ziegler Nichols method may determine very precise parameters for controller, it also can fine-tune after this.

\section{1 Ziegler Nichols PID parameter setting method:}

(1)Build a closed loop control circuit, determine the stability limit.

(2) According to the formula to calculate the controller parameters.

The stability limit is determined by the P component. When there is a steady oscillation to reach this limit, the critical coefficient Kpcrit and critical oscillation cycle Tcrit are generated. After determining the critical coefficient Kpcrit and critical oscillation period Tcrit, we calculate other parameters according to a formula in the Table 1.

According to the experience formula of following table and the corresponding controller type set corresponding PID parameters, and then conduct simulation validation and fine-tuning:

Table 1 PID parameters calculating formula

\begin{tabular}{|c|c|c|c|c|c|}
\hline \multirow{2}{*}{$\begin{array}{c}\text { The controller } \\
\text { type }\end{array}$} & \multicolumn{5}{|c|}{ Characteristic data } \\
\cline { 2 - 6 } & $K_{P}$ & $T_{I}$ & $T_{D}$ & $K_{i}$ & $K_{D}$ \\
\hline PID & $0.6 K_{\text {Pcrit }}$ & $0.5 T_{\text {crit }}$ & $0.12 T_{\text {crit }}$ & $\frac{K_{P}}{T_{I}}$ & $K_{P} T_{D}$ \\
\hline
\end{tabular}

For the transfer function of a given controlled object, we select the intersection point between root locus diagram of discrete system and z plane unit circle on the root locus diagram, then we gain $\mathrm{Km}$ and this point $\omega$ value namely $\omega_{m}$. Setting formula is as follows:

$$
K_{p}=0.6 K_{m}, \quad K_{d}=\frac{K_{p} \pi}{4 \omega_{m}}, K_{i}=\frac{K_{p} \omega_{m}}{\pi}
$$

In formula, $\mathrm{Km}$ is the $\mathrm{Kp}$ values of the system initiate oscillation, $\omega_{m}$ for the oscillation frequency. Oscillation frequency $\omega_{m}$ can be determined by the pole Angle $\theta$ located on the unit circle, $\omega_{m}=\theta / \mathrm{T}$ ( $\mathrm{T}$ for the sampling period $)$.

\subsection{The Matlab setting program}

Take sampling period: $\mathrm{T}=0.25 \mathrm{~s}$; the amplifier amplification coefficient: $\mathrm{Kp}=50$. The operation result is shown in Figure 1. 


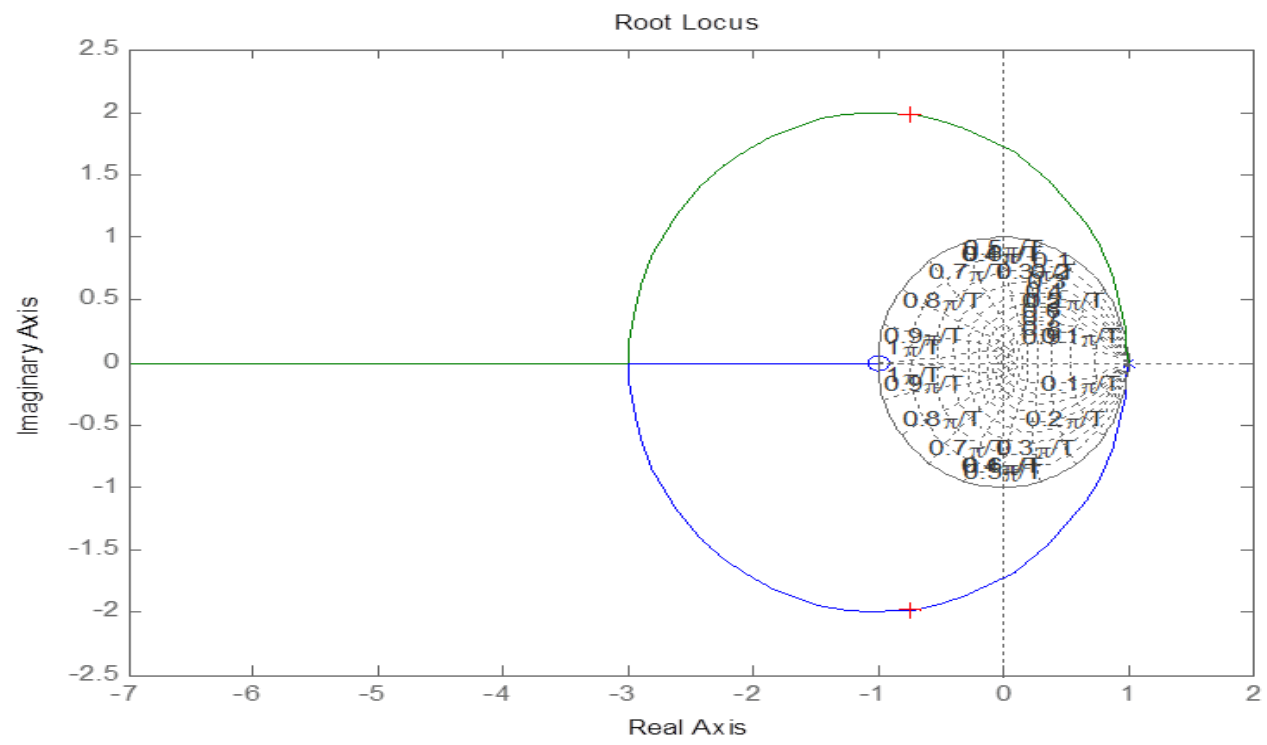

Fig.1. Root locus diagram of program

Select a point in the graphics window.

$\mathrm{km}=5.7977 \mathrm{e}+07$

pole $=-0.7344+1.9818 \mathrm{i}$

$-0.7344-1.9818 \mathrm{i}$

$\mathrm{kp}=3.4786 \mathrm{e}+07$

$\mathrm{kd}=3.5469 \mathrm{e}+06$

$\mathrm{ki}=8.5292 \mathrm{e}+07$

\section{The Simulink Simulation}

\subsection{To construct a system of Simulink model}

Based on the analysis of the mechanical theory as the foundation, designed the soccer robot pick the ball institutions optimal design process[9] [10], found aim function, select design variables and the corresponding optimization algorithm to optimize a complete set of institutions. At last through the test the final performance parameters of the institution are obtained. Experiments show that the system has higher accuracy and stability, the new optimize pick the ball have design basic requirements, and achieved good ideal control effect.

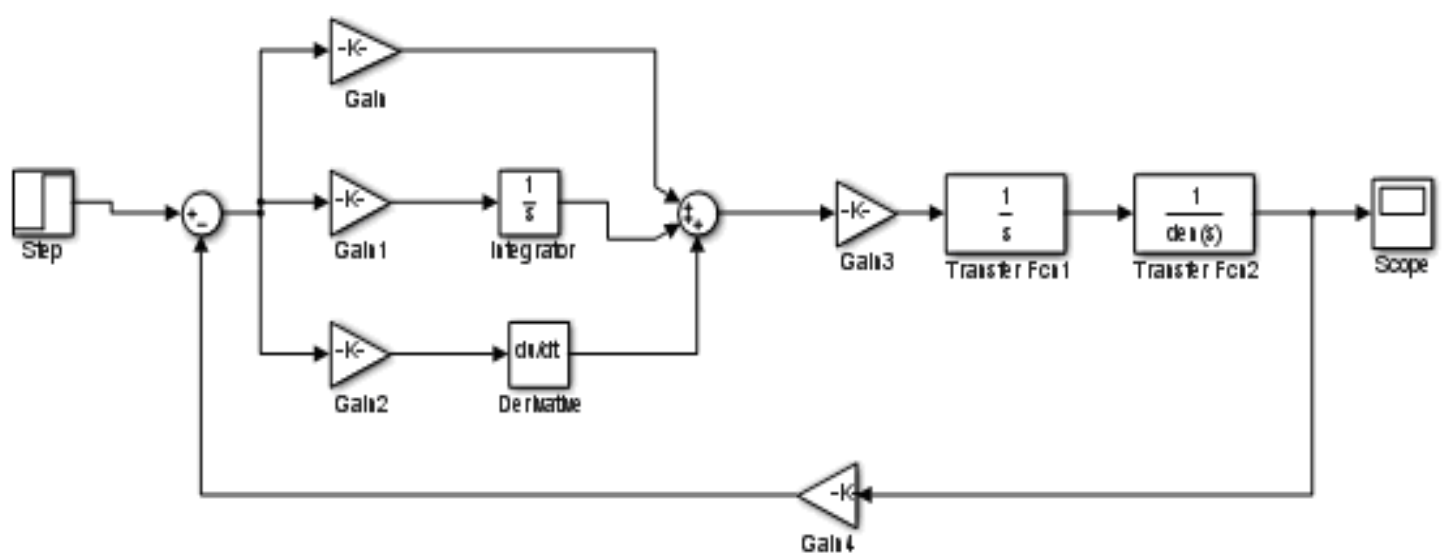

Fig.2 System simulation model 


\subsection{The results of Simulation}

The experimental results show that the system has a good response speed, stability and accuracy, and strong robustness.

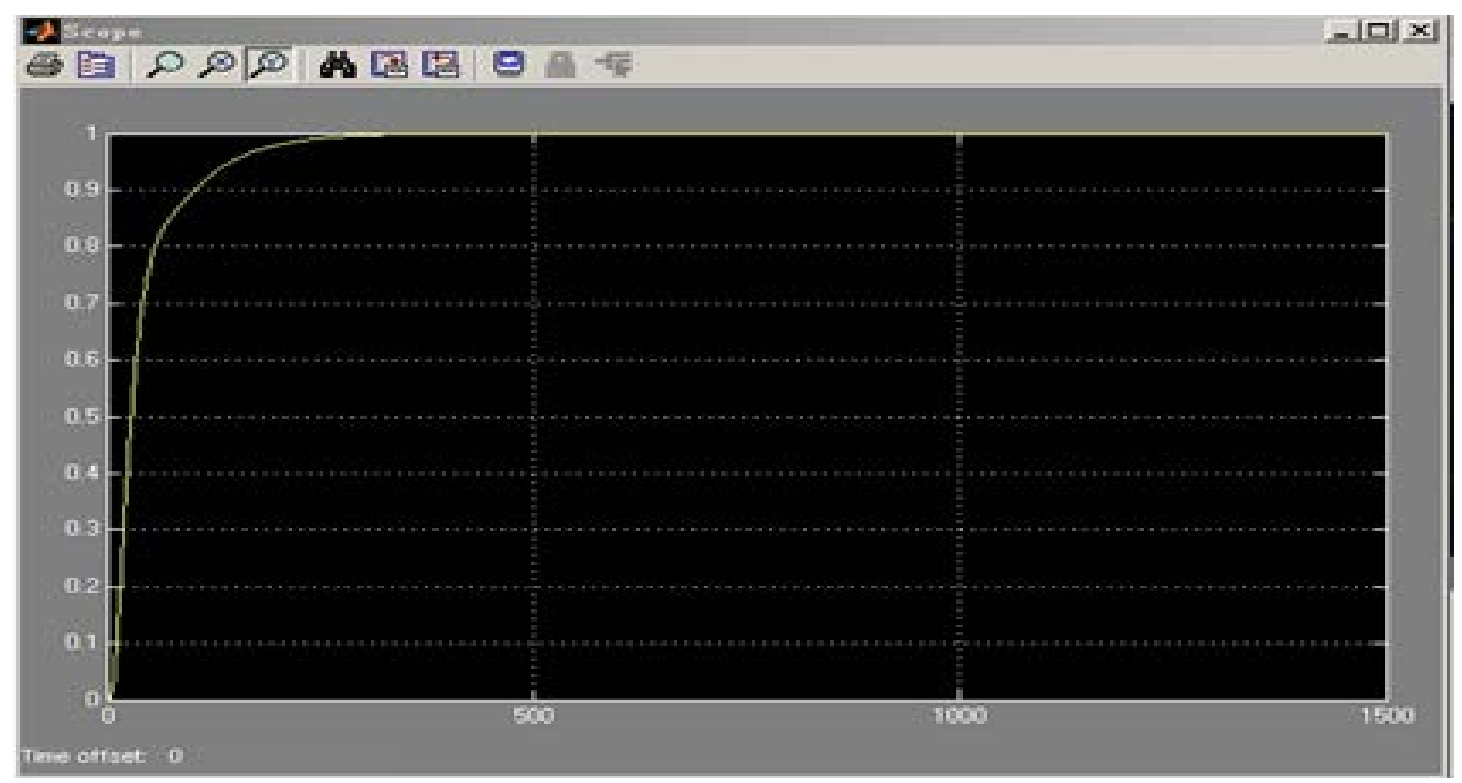

Fig.3 Adaptive PID dynamic response based on Ziegler Nichols - frequency method

\section{Conclusion}

In a closed loop control system, the integral control link improves the steady-state error accuracy of the system. Due to the constantly accumulated error, it can eliminate the error quickly, but it make the system produces overshoot; And differential control can increase the damping of the system and improve the dynamic response speed. Because the PID controller can compensate the vast majority of the control system, the setting method is simple and good robustness; therefore it is currently the most widely used controller. Ziegler-Nichols methods are method of PID setting based on system stability analysis. Ziegler-Nichols method can quickly and accurately calculate the corresponding parameter numerical, then only need to fine-tune the ideal control effect can be got.

By Ziegler Nichols - frequency method, we get a set of parameters for the $\mathrm{Kp}=3.4786 \mathrm{e}+07$; Kd $=3.5469 \mathrm{e}+06 ; \mathrm{Ki}=8.5292 \mathrm{e}+07$. According to the above parameters, the system has finally a high control precision, faster response speed, more outstanding control stability, almost completely resistant to outside interference.

This method can realize the PID controller adaptive and ensure the control loop always work in the best condition even under the operating conditions change. It is of great significance for industrial enterprises the energy-saving emission reduction and improving the operation efficiency of process equipment.

\section{Reference}

[1]Ming-Hsun Chiang, Fan-Ren Chang, Anthropomorphic Design of the Human-Like Walking Robot[J], Journal of Bionic Engineering,2013:10(2),186-193.

[2]Chung Yik Lau, Almon Chai, The Development of a Low Cost Pneumatic Air Muscle Actuated Anthropomorphic Robotic Hand[J], Procedia Engineering,2012(41), 737-742.

[3]F.J. Brosed, J. Santolaria, J.J. Aguilar, D. Guillomía, Laser triangulation sensor and six axes anthropomorphic robot manipulator modelling for the measurement of complex geometry products[J], Robotics and Computer-Integrated Manufacturing, 2012:28(6), 660-671. 
[4]MirSaleh Bahavarnia, Mohammad Saleh Tavazoei, A new view to Ziegler-Nichols step response tuning method: Analytic non-fragility justification[J], Journal of Process Control, 2013:23(1), 23-33.

[5]Tomislav B. Šekara, Miroslav R. Mataušek, Revisiting the Ziegler-Nichols process dynamics characterization, [J] Journal of Process Control, 2010:20(3), 360-363.

[6] Tang Jie, Motor and Drag (second edition) [M], higher education press, 2007.

[7]Gu Deying, Computer Control technology (3rd edition) [M], Beijing university of posts and telecommunications, 2012.

[8]Chen Boshi, Electric Drive Automatic Control System [M], mechanical industry publishing house,2011.

[9]Zhao Jingbo, MATLAB Simulation and Control System Design[M], mechanical industry publishing house, 2010.

[10]Liu Jinkun, Advanced PID Control MATLAB Simulation[M], the electronic industry press,2011. 